\title{
Interactive comment on "Detection and origin of different types of annual laminae in recent stalagmites from Zoolithencave, southern Germany: Evaluation of the potential for quantitative reconstruction of past precipitation variability" by D. F. C. Riechelmann et al.
}

Anonymous Referee \#1

Received and published: 12 April 2016

Some general comments:

The authors present a study of modern stalagmites from an intersting cave site in Germany also known for its archeological and paleontological remains. Studying various types of lamination patterns in speleothems and other paleoclimate archives is undoubtedly important in order to establish robust high-resolution connections to climate relevant variables. In this regard the manuscript is relevant to various climate studies published in journals as "Climate of the Past".

Printer-friendly version

Discussion paper

(c) (i) 
In the manuscript a comparison of petrographic and elemental evidence to meteorological data is provided. In its current form, however, the results and discussion are not really bridging the gap from proxy data to instrumental meteorological and numeric climate data. Envisaging a "quantitative reconstruction" based on proxy evidence is always challenging and with regard to speleothems might only be reliable in connection with a deeper understanding of the actual cave/speleothem vs. climate relationships, i.e. also based on results from high-resolution cave monitoring. Such data and relationships are not presented in the manuscript and consequently some of the explanations given are still of some qualitative character.

The stalagmite samples presented are difficult with regard to establishing a U-Th based chronology (due to detrital contamination). An approach at its best is provided via an indirect age constraint based on radiocarbon dating of charcoal and partially detecting the evolution of $14 \mathrm{C}$ bomb peaks. Altogether, however, the chronology (being a critical issue regarding the intention of this study) is not robust in a stricter sense making the annual nature of the lamination and some of the relations discussed a subject to debate.

Of interest and high relevance in this study are the various statistical methods of variable complexity being applied to the datasets, e.g. methods typically applied in treering research. Although all of these methods are well-known and might be (widely) accepted, the critical reader encounters some flavor of significant and sometimes subjective (e.g. inserting specific numbers of laminae, timing of $14 \mathrm{C}$ bomb peak evolution) tuning being applied. Some scientists will consider this problematic regarding the reliability of the actual proxy-climate relationships and there are controversial and ongoing discussions on that topic. In some speleothem records the connections proposed are clearly more obvious, i.e. without sophisticated statistical treatment.

Considering the origin and rhythm of the lamination observed and some of the parameter relationships the discussion should me more critical and diverse and also open to possible alternative explanations. Some of the conclusions drawn seem too simple 
based on the chronology and data presented.

Particular chapters/paragraphs/lines:

Introduction: Informative and comprehensive, e.g. regarding the broad literature cited.

Line 60ff: Regarding the various lamina types the frequent occurrence of "event lamina-

Interactive tion" could also be mentioned, e.g. lamination resulting from flooding of cave chambers (clay layers) being a potential proxy for high rainfall and floods in continental areas or lamination in some coastal caves representing sea-level fluctuations (e.g. Antonioli et al., 2004; Dorale et al., 2010).

Dating methods: Lines 131f: Why do the authors not use the updated half-lives reported in Cheng et al. (2013, EPSL)?

No U-Th data are available from stalagmite Zoo-rez-2. Maybe this stalagmite is significantly cleaner (less detrital contamination) and/or the $U$ contents are higher. The petrography looks somewhat lighter in color (Fig. 3), so the stalagmite might be cleaner eventually. Might be worth trying.

Visible laminae: This is a critical issue. Based on the microscopic pictures shown (Fig. 4; $2 \mathrm{~mm}$ scale bar!) some of the clear (annual) layers look much thicker (up to $\mathrm{mm}$-scale) than supported by the average lamina thickness calculations, i.e. the visual inspection is not consistent with the calculated thicknesses (few hundreds of microns). Sometimes brownish layers are very close to each other and even look like very thin doubled brownish layers (cf. Baker et al., 2002) followed by thick clear layers. Based on visual inspection the lamina development looks quite unregular in particular with regard to lamina thickness and it could even be some kind of event lamination.

Considering the absolute lamina counts from the parallel tracks of Zoo-rez-1 (124 vs. 161 vs. 135; lines 201ff) these (small) numbers are relatively far from each other despite their proximity within the same stalagmite. This should be addressed more critically. 
Suggesting visible annual laminae the authors should provide some more direct (microscopic) comparison of, e.g. petrography vs. selected trace elements within a typical stalagmite section. This is frequently provided in speleothem lamination studies and gives more confidence regarding high-resolution correlations.

Chronology of stalagmite Zoo-rez: The 14C-dating of a basal charcoal piece might be considered a minimum age constraint but does not necessarily constrain the absolute age of initial stalagmite formation - in particular when reviewing the position of the charcoal piece, i.e. significantly below initial stalagmite growth (cf. Fig. 3). A significant amount of sedimentation time might have passed between $14 \mathrm{C}$ age and start of lamination development and therefore it is not a direct age constraint compared to U-Th data.

A calibrated 1-sigma dating range of 1671-1951 AD is reported for the single radiocarbon age (line 230). This is a relatively large range and therefore only moderate constraint of the initial growth. It should be addressed more critically.

Regarding the detection of the $14 \mathrm{C}$ bomb peak(s): Information on radiocarbon in speleothems is given well. In order to capture the timing of decrease in atmospheric radiocarbon activity, however, some additional $14 \mathrm{C}$ data would be needed to (better) constrain the timing of growth cessation. "Since the increase in 14C in Zoo-rez is large (compare e.g., Noronha et al., 2015), the peak is near the maximum of the atmospheric $14 \mathrm{C}$ values." (lines 243f). Based on the data points presented in Fig. 5 this seems rather speculative and in fact not a strong anchor for the claimed (narrow) timing of the stalagmite top (1970 AD +- 5 years; cf. lines $245 \mathrm{ff}$ ). The upper limit of $14 \mathrm{C}$ activity in drip water from this cave site might be significanlty higher before decreasing to lower values.

"Zoo-rez did not grow until 1999 AD" (year of sampling; cf. lines 239f). Instead, growth cessation in $1970 \mathrm{AD}$ is suggested. This is strange considering that the stalagmite was fed by an active drip suggesting recent growth (lines 120f). Why is the drip water not

CPD

Interactive comment
Printer-friendly version

Discussion paper 
supersaturated? Why is the system potentially disturbed?

Elemental laminae A comparison of the modern stalagmite composition with modern hydrochemical data would be of high value in order to establish robust relationships, e.g. annual variability of elemental supply.

Procedure and interpretation of wavelet analysis shown in Fig. 8: Provide more explanation!

lines 270ff: The correspondance of $\mathrm{Mg}$ concentration and lamina thickness does not prove the annual nature of the lamination pattern, only some possible interrelation between the two parameters. However, this interrelation should also be supported by some more direct comparison.

$\mathrm{Al}$ and $\mathrm{Mn}$ concentrations are considered proxies for detrital content in the stalagmites. What about silicon? This element is most typical for siliciclastic input, e.g. clay minerals, feldspars.

Interpretation and Discussion

Chronology lines 300ff: A corresponding number of missing laminae was inserted into the chronology. 124 vs. 161 counted laminae constitute a significant mismatch. Moreover, lamina thickness was assumed for insertion. So, nine laminae were inserted into the "master track". The reader gets an impression of playing around, i.e. a semiquantitative and somewhat arbitrary approach. Provide more information and argumentation with regard to this procedure!

lines 315ff: Please explain meaning and significance of the correlation procedure and values!

lines 327ff: Regarding the lamina adaptions and radiocarbon age constraints: See comments above. Altogether it seems a somewhat floating chronology.

lines 332: A critical issue. Cave ventilation is excluded by the authors regarding lam-
CPD

Interactive

comment
Printer-friendly version

Discussion paper 
ina development and measured (monitored) cave pCO2 values. However, an annual amplitude of around 1000 ppmv in pCO2 is relatively large, i.e. more than twice the absolute atmospheric concentration. Moreover, the obviously higher values are "removed" seasonally approaching atmospheric compositions during wintertime. Also, the stalagmites were collected from the entrance section being more prone to influences of cave air exchange. Consequently, there are several major arguments for cave ventilation playing a potential role. Excluding such effects in your interpretation is too simple in this particular case. The authors further state: "...stalagmite growth is dominated by the winter season" (lines 502f). This is frequently the case in seasonally ventilated cave systems of the mid latitudes, i.e. another argument for cave ventilation most likely playing a significant role for speleothem deposition.

lines 337f: Annual lamination in stalagmite Zoo-rez is related to annual changes in drip water composition. Give more information and explanation! What kind of changes? Are monitoring data available/published?

Fig. 9 shows a strong variation in annual lamina thickness. This looks interesting. Is there some multi-annual cyclicity represented?

Wiggle matching and data interpolation lines 357f: The shifts performed during the wiggle matching procedure are up to several typical lamina-thicknesses. So, particular numbers of laminae are inserted and subsequently significantly shifted. This is critical regarding a robust chronology and data interpretation.

lines 366f: "The clear layer corresponds to higher Mg concentration and the brownish pigmented to lower Mg concentration." This seems mainly based on wiggle matching. It should be shown easily by a direct comparison of Mg concentration vs. stalagmite petrography on a microscopic scale (visually), i.e. the resolution is high enough in both proxies.

Fig. 12: After sophisticated tuning treatments a direct comparison from year to year is still difficult. There is an increasing trend in lamina thickness and $\mathrm{Mg}$ - this is interesting

Interactive comment

Printer-friendly version

Discussion paper 
and might be worth of further discussion.

Interpretation of proxy signals in terms of past climate variability lines $385 \mathrm{ff}$ : As already mentioned, the seasonal relations proposed would be more robust in combination with some hydrochemical data.

Fig. 13: Some anticorrelation of $\mathrm{Mg}$ and $\mathrm{Y}$. This deserves more discussion/explanation.

Does some positive correlation of increased $\mathrm{Sr}$, Ba with thicker laminae exist? Some studies relate the $\mathrm{Sr}$, Ba incorporation to carbonate precipitation rates (e.g. Lorens, 1981; Tesoriero and Pankow, 1996; Huang and Fairchild, 2001).

UV-luminescence The brownish layers probably depend on humic- and fulvic acids from the soil (constraint from UV luminescence). However, these components could also be introduced during snowmelt flushing events. As mentioned, in the microscopic image some of the brownish layers look like a double-layer potentially respresenting two events close to each other, e.g. snowmelts.

line 464: Fig. 14 (not 13).

lines 465: The authors talk about "detrital" material/contamination. What kind of detritus? Being siliciclastic material Si and Al should be typically high. If $\mathrm{Mn}$ is high then I would also expect Fe being relatively high. The form and origin of detrital contamination is not constrained well and therefore somewhat speculative.

Lamina thickness Fig. 15: Some correspondance but also some mismatch (earlier part).

References: Informative and extensive. A broad range of the literature available on that topic is represented.

Finally: Considering the interesting and paleoclimatically relevant topic, as well as broad approach involving various analytical and statistical techniques being potentially helpful to other high-resolution studies, I suggest publication after some major revi-

\section{CPD}

comment
Printer-friendly version

Discussion paper 
sions.

In particular, a more critical interpretation of the results and more diverse discussion is CPD suggested. The authors should seek for going a step further compared to the already existing and increasing number of studies being conducted on that topic, i.e. a more quantitative connection to climate variability of the recent past in the region studied. In this context, I strongly suggest some combination with cave monitoring results making the story (and in particular the proxy dependencies) more reliable.

Interactive comment on Clim. Past Discuss., doi:10.5194/cp-2016-18, 2016.

Interactive

comment

Discussion pap 